\title{
Gravitational waves from hot young rapidly rotating neutron stars
}

\author{
Benjamin J. Owen, ${ }^{1}$ Lee Lindblom, ${ }^{1}$ Curt Cutler, ${ }^{2}$ Bernard F. Schutz, ${ }^{2}$ Alberto Vecchio, ${ }^{2}$ and Nils Andersson ${ }^{3}$ \\ ${ }^{1}$ Theoretical Astrophysics 130-33, California Institute of Technology, Pasadena, California 91125 \\ ${ }^{2}$ Max Planck Institute for Gravitational Physics, Schlaatzweg 1, D-14473 Potsdam, Germany \\ ${ }^{3}$ Institute for Astronomy and Astrophysics, University of Tübingen, D-72076 Tübingen, Germany
}

(Received 20 April 1998; published 14 September 1998)

\begin{abstract}
Gravitational radiation drives an instability in the $r$-modes of young rapidly rotating neutron stars. This instability is expected to carry away most of the angular momentum of the star by gravitational radiation emission, leaving a star rotating at about $100 \mathrm{~Hz}$. In this paper we model in a simple way the development of the instability and evolution of the neutron star during the year-long spindown phase. This allows us to predict the general features of the resulting gravitational waveform. We show that a neutron star formed in the Virgo cluster could be detected by the LIGO and VIRGO gravitational wave detectors when they reach their "enhanced" level of sensitivity, with an amplitude signal-to-noise ratio that could be as large as about 8 if near-optimal data analysis techniques are developed. We also analyze the stochastic background of gravitational waves produced by the $r$-mode radiation from neutron-star formation throughout the universe. Assuming a substantial fraction of neutron stars are born with spin frequencies near their maximum values, this stochastic background is shown to have an energy density of about $10^{-9}$ of the cosmological closure density, in the range $20 \mathrm{~Hz}$ to $1 \mathrm{kHz}$. This radiation should be detectable by "advanced" LIGO as well. [S0556-2821(98)03616-9]

PACS number(s): 04.30.Db, 04.40.Dg, 97.60.Jd
\end{abstract}

\section{INTRODUCTION}

Recently Andersson [1] discovered that gravitational radiation tends to destabilize the $r$-modes of rotating stars. Friedman and Morsink [2] then showed that this instability is generic, in the sense that gravitational radiation tends to make all $r$-modes in all rotating stars unstable. Lindblom, Owen, and Morsink [3] have recently evaluated the timescales associated with the growth of this instability. Gravitational radiation couples to these modes through the current multipoles rather than the more typical mass multipole moments. This coupling is stronger than anyone anticipated for these modes, and is so strong in fact that the viscous forces present in hot young neutron stars are not sufficient to suppress the gravitational radiation driven instability. Gravitational radiation is expected therefore to carry away most of the angular momentum of hot young neutron stars. These results have now been verified by Andersson, Kokkotas, and Schutz [4].

In this paper we study the gravitational waveforms that are produced as the $r$-mode instability grows and radiates away the bulk of the angular momentum of a hot young rapidly rotating neutron star. The properties of the $r$-modes and the instability associated with them are reviewed in Sec. II. The equations that describe (approximately) the evolution of the $r$-modes as they grow and spin down a rapidly rotating neutron star are derived in Sec. III. These equations are solved numerically and the results are also presented in Sec. III. The gravitational waveforms associated with the $r$-mode instability are evaluated in Sec. IV. General analytical and detailed numerical expressions for these waveforms are presented. In Sec. V we evaluate the detectability of this type of gravitational wave signal by the laser interferometer gravitational wave detectors such as LIGO [5], VIRGO [6], and GEO [7]. We consider the detectability of signals produced by single nearby sources, and also the detectability of a sto- chastic background of sources from throughout the universe. Finally, in Sec. VI we discuss the prospects for gravitationalwave astronomy opened up by the $r$-modes.

\section{THE $R$-MODE INSTABILITY}

The $r$-modes of rotating Newtonian stars are generally defined to be solutions of the perturbed fluid equations having (Eulerian) velocity perturbations of the form

$$
\delta \vec{v}=R \Omega f(r / R) \vec{Y}_{l m}^{B} e^{i \omega t},
$$

where $R$ and $\Omega$ are the radius and angular velocity of the unperturbed star, $f(r / R)$ is an arbitrary dimensionless function, and $\vec{Y}_{l m}^{B}$ is the magnetic type vector spherical harmonic defined by

$$
\vec{Y}_{l m}^{B}=[l(l+1)]^{-1 / 2} r \vec{\nabla} \times\left(r \vec{\nabla} Y_{l m}\right) .
$$

For barotropic stellar models, of primary concern to us here, the Euler equation determines the form of these modes: the radial dependence $f(r / R)$ is determined to be $f(r / R)$ $=\alpha(r / R)^{l}$, where $\alpha$ is an arbitrary constant [8]. These modes exist with velocity perturbations as given by Eq. (2.1) if and only if $l=m$ [8]. Also, the frequencies of these modes are given by [9]

$$
\omega=-\frac{(l-1)(l+2)}{l+1} \Omega .
$$

These modes represent large scale oscillating currents that move (approximately) along the equipotential surfaces of the rotating star. The restoring force for these oscillations is the Coriolis force; hence the frequencies of these modes are low compared to the usual $f$ and $p$-modes in slowly rotating stars. These expressions for $\delta \vec{v}$ and $\omega$ are the lowest order 
terms in an expansion in terms of the angular velocity $\Omega$. The exact expressions contain additional terms of order $\Omega^{3}$. There may exist other modes of rotating barotropic stellar models with properties similar to these classical $r$-modes; however, our discussion here is limited to the properties of these classical $r$-modes.

The density perturbation associated with the $r$-modes can be deduced by evaluating the inner product of $\vec{v}$ (the unperturbed fluid velocity) with the perturbed Euler equation, and the equation for the perturbed gravitational potential [10]:

$$
\begin{aligned}
\delta \rho= & \alpha R^{2} \Omega^{2} \rho \frac{d \rho}{d p}\left[\frac{2 l}{2 l+1} \sqrt{\frac{l}{l+1}}\left(\frac{r}{R}\right)^{l+1}+\delta \Psi(r)\right] \\
& \times Y_{l+1 l} e^{i \omega t} .
\end{aligned}
$$

The quantity $\delta \Psi$ is proportional to the perturbed gravitational potential $\delta \Phi$, and is the solution to the ordinary differential equation

$$
\begin{gathered}
\frac{d^{2} \delta \Psi(r)}{d r^{2}}+\frac{2}{r} \frac{d \delta \Psi(r)}{d r}+\left[4 \pi G \rho \frac{d \rho}{d p}-\frac{(l+1)(l+2)}{r^{2}}\right] \delta \Psi(r) \\
=-\frac{8 \pi G l}{2 l+1} \sqrt{\frac{l}{l+1}} \rho \frac{d \rho}{d p}\left(\frac{r}{R}\right)^{l+1}
\end{gathered}
$$

which satisfies appropriate asymptotic boundary conditions. We note that $\delta \rho$ is proportional to $\Omega^{2}$ and hence is small (i.e., higher order in $\Omega$ ) compared to $\delta \vec{v}$ in slowly rotating stars. We also note that $\delta \rho$ is proportional to $Y_{l+1 l}$-having spherical harmonic index one order in $l$ higher than that of the velocity perturbation. Equation (2.4) is the complete expression for the density perturbation to order $\Omega^{2}$. The exact expression for $\delta \rho$ includes additional terms of order $\Omega^{4}$.

The $r$-modes evolve with time dependence $e^{i \omega t-t / \tau}$ as a consequence of ordinary hydrodynamics and the influence of the various dissipative processes. The real part of the frequency of these modes, $\omega$, is given in Eq. (2.3), while the imaginary part $1 / \tau$ is determined by the effects of gravitational radiation, viscosity, etc. The simplest way to evaluate $1 / \tau$ is to compute the time derivative of the energy $\widetilde{E}$ of the mode (as measured in the rotating frame). $\widetilde{E}$ can be expressed as a real quadratic functional of the fluid perturbations:

$$
\widetilde{E}=\frac{1}{2} \int\left[\rho \delta \vec{v} \cdot \delta \vec{v}^{*}+\left(\frac{\delta p}{\rho}-\delta \Phi\right) \delta \rho^{*}\right] d^{3} x
$$

Thus the time derivative of $\widetilde{E}$ is related to the imaginary part of the frequency $1 / \tau$ by

$$
\frac{d \widetilde{E}}{d t}=-\frac{2 \widetilde{E}}{\tau} .
$$

Since the specific expressions for the time derivative of $\widetilde{E}$ due to the influences of gravitational radiation [11] and viscosity [12] are well known, Eq. (2.7) may be used to evaluate the imaginary part of the frequency.
It is convenient to decompose $1 / \tau$ :

$$
\frac{1}{\tau(\Omega)}=\frac{1}{\tau_{G R}(\Omega)}+\frac{1}{\tau_{S}(\Omega)}+\frac{1}{\tau_{B}(\Omega)},
$$

where $1 / \tau_{G R}, 1 / \tau_{S}$, and $1 / \tau_{B}$ are the contributions due to gravitational radiation emission, shear viscosity and bulk viscosity, respectively. Expressions for these individual contributions for the $r$-modes are given by [3]

$$
\begin{aligned}
& \frac{1}{\tau_{G R}}=-\frac{32 \pi G \Omega^{2 l+2}}{c^{2 l+3}} \frac{(l-1)^{2 l}}{[(2 l+1) ! !]^{2}}\left(\frac{l+2}{l+1}\right)^{2 l+2} \\
& \times \int_{0}^{R} \rho r^{2 l+2} d r \\
& \frac{1}{\tau_{S}}=(l-1)(2 l+1) \int_{0}^{R} \eta r^{2 l} d r\left(\int_{0}^{R} \rho r^{2 l+2} d r\right)^{-1}
\end{aligned}
$$

and

$$
\frac{1}{\tau_{B}} \approx \frac{4 R^{2 l-2}}{(l+1)^{2}} \int \zeta\left|\frac{\delta \rho}{\rho}\right|^{2} d^{3} x\left(\int_{0}^{R} \rho r^{2 l+2} d r\right)^{-1},
$$

where $\delta \rho$ is given in Eq. (2.4). We note that the expression for $1 / \tau_{B}$ in Eq. (2.11) is only approximate. The exact expression should contain the Lagrangian density perturbation $\Delta \rho$ in place of the Eulerian perturbation $\delta \rho$. The bulk viscosity [see Eq. (2.13)] is a very strong function of the temperature, being proportional to $T^{6}$. Thus, the result of any error that might occur in our approximation for $1 / \tau_{B}$ is simply to shift slightly the temperature needed to achieve a given viscosity timescale. Numerical estimates show that changing this quantity by even a factor of one hundred (as suggested by Ref. [4]), does not substantially affect the important physical quantities computed here (i.e. the spindown rate or the final angular velocity of the star).

We have evaluated these expressions for the imaginary parts of the frequency for a "typical" neutron star model with a polytropic equation of state: $p=k \rho^{2}$, with $k$ chosen so that a $1.4 M_{\odot}$ model has the radius $12.53 \mathrm{~km}$. We use the usual expressions for the viscosity of hot neutron star matter [13]:

$$
\begin{aligned}
& \eta=347 \rho^{9 / 4} T^{-2}, \\
& \zeta=6.0 \times 10^{-59}\left(\frac{l+1}{2 \Omega}\right)^{2} \rho^{2} T^{6},
\end{aligned}
$$

where all quantities are expressed in cgs units. We have evaluated the expressions, Eqs. (2.9)-(2.11), for the dissipative timescales with fiducial values of the angular velocity $\Omega=\sqrt{\pi G \bar{\rho}}$ and temperature $T=10^{9} \mathrm{~K}$. These fiducial timescales $\tilde{\tau}_{G R}, \tilde{\tau}_{V}$, and $\tilde{\tau}_{B}$ are given in Table I for the $r$-modes with $2 \leqslant l \leqslant 6$. It will be useful in the following to define a timescale associated with the viscous dissipation $1 / \tau_{V}=1 / \tau_{S}+1 / \tau_{B}$. The viscous timescale $\tau_{V}$ and the gravi- 
TABLE I. Gravitational radiation and viscous timescales (in seconds) are presented for $T=10^{9} \mathrm{~K}$ and $\Omega=\sqrt{\pi G \bar{\rho}}$.

\begin{tabular}{cccc}
\hline \hline$l$ & $\tilde{\tau}_{G R}$ & $\tilde{\tau}_{S}$ & $\tilde{\tau}_{B}$ \\
\hline 2 & $-3.26 \times 10^{0}$ & $2.52 \times 10^{8}$ & $6.99 \times 10^{8}$ \\
3 & $-3.11 \times 10^{1}$ & $1.44 \times 10^{8}$ & $5.13 \times 10^{8}$ \\
4 & $-2.85 \times 10^{2}$ & $1.07 \times 10^{8}$ & $4.01 \times 10^{8}$ \\
5 & $-2.37 \times 10^{3}$ & $8.79 \times 10^{7}$ & $3.26 \times 10^{8}$ \\
6 & $-1.82 \times 10^{4}$ & $7.58 \times 10^{7}$ & $2.74 \times 10^{8}$ \\
\hline \hline
\end{tabular}

tational timescale $\tau_{G R}$ can be expressed then in terms of the fiducial timescales in a way that makes their temperature and angular velocity dependences explicit:

$$
\begin{aligned}
& \frac{1}{\tau_{V}}=\frac{1}{\tilde{\tau}_{S}}\left(\frac{10^{9} \mathrm{~K}}{T}\right)^{2}+\frac{1}{\tilde{\tau}_{B}}\left(\frac{T}{10^{9} \mathrm{~K}}\right)^{6}\left(\frac{\Omega^{2}}{\pi G \bar{\rho}}\right), \\
& \frac{1}{\tau_{G R}}=\frac{1}{\tilde{\tau}_{G R}}\left(\frac{\Omega^{2}}{\pi G \bar{\rho}}\right)^{l+1} .
\end{aligned}
$$

\section{EVOLUTION OF THE $R$-MODES}

To determine the gravitational waveform that will result from the instability in the $r$-modes, we must estimate how the neutron star evolves as the instability grows and radiates the angular momentum of the star away to infinity. Initially the mode will be a small perturbation that is described adequately by the linear analysis that we have described above. However, as the mode grows, non-linear hydrodynamic effects become important and eventually dominate the dynamics. At the present time we do not have available the tools to follow exactly this non-linear phase of the evolution. Instead, we propose a simple approximation that includes (we believe) the basic features of the exact evolution.

We treat the star as a simple system having only two degrees of freedom: the uniformly rotating equilibrium state parametrized by its angular velocity $\Omega$, and the $r$-mode parametrized by its amplitude $\alpha$. The total angular momentum $J$ of this simple model of the star is given by,

$$
J=I \Omega+J_{c},
$$

where $I$ is the moment of inertia of the equilibrium state of the star, and $J_{c}$ is the canonical angular momentum of the $r$-mode.

In this simple model of the star the angular momentum $J$ is a function of the two parameters that characterize the state of the system: $J=J(\Omega, \alpha)$. We can determine this functional relationship approximately as follows. The canonical angular momentum of an $r$-mode can be expressed in terms of the velocity perturbation $\delta \vec{v}$ by [14],

$$
J_{c}=-\frac{l}{2(\omega+l \Omega)} \int \rho \delta \vec{v} \cdot \delta \vec{v} * d^{3} x .
$$

For the $l=2 r$-mode of primary interest to us here this expression reduces (at lowest order in $\Omega$ ) to

$$
J_{c}=-\frac{3}{2} \Omega \alpha^{2} \widetilde{J} M R^{2},
$$

where $\widetilde{J}$ is defined by

$$
\widetilde{J}=\frac{1}{M R^{4}} \int_{0}^{R} \rho r^{6} d r .
$$

For the polytropic models studied in detail here the dimensionless constant $\widetilde{J}=1.635 \times 10^{-2}$. The moment of inertia $I$ can also be conveniently expressed as

$$
I=\widetilde{I} M R^{2},
$$

where $\widetilde{I}$ is given by

$$
\widetilde{I}=\frac{8 \pi}{3 M R^{2}} \int_{0}^{R} \rho r^{4} d r .
$$

For the polytropic models considered here $\widetilde{I}=0.261$. Thus, our simple model of the angular momentum of the perturbed star is

$$
J(\Omega, \alpha)=\left(\widetilde{I}-\frac{3}{2} \widetilde{J} \alpha^{2}\right) \Omega M R^{2} .
$$

The perturbed star loses angular momentum primarily through the emission of gravitational radiation. Thus, we compute the evolution of $J(\Omega, \alpha)$ by using the standard multipole expression for angular momentum loss. The $l=2$ $r$-mode is the primary source of gravitational radiation in our simple model of this system, and this mode loses angular momentum primarily through the $l=m=2$ current multipole. Thus the angular momentum of the star evolves as

$$
\frac{d J}{d t}=-\frac{c^{3}}{16 \pi G}\left(\frac{4 \Omega}{3}\right)^{5}\left(S_{22}\right)^{2}
$$

The $l=m=2$ current multipole moment $S_{22}$ for this system is given by

$$
S_{22}=\sqrt{2} \frac{32 \pi}{15} \frac{G M}{c^{5}} \alpha \Omega R^{3} \widetilde{J} .
$$

Combining Eq. (3.8) for the angular momentum evolution of the star with Eqs. (2.9) and (3.7), we obtain one equation for the evolution of the parameters $\Omega$ and $\alpha$ that determine the state of the star:

$$
\left(\widetilde{I}-\frac{3}{2} \alpha^{2} \widetilde{J}\right) \frac{d \Omega}{d t}-3 \alpha \Omega \widetilde{J} \frac{d \alpha}{d t}=\frac{3 \alpha^{2} \Omega \widetilde{J}}{\tau_{G R}} .
$$

During the early part of the evolution of the star, the perturbation analysis of the $r$-modes described earlier applies. In addition to radiating angular momentum from the star via gravitational radiation, the mode will also lose energy via gravitational radiation and neutrino emission (from the bulk viscosity) and also deposit energy into the thermal state of the star due to shear viscosity. It is most convenient to obtain the equation for the energy balance during this part 
of the evolution in terms of the energy $\widetilde{E}$ of the mode as defined in Eq. (2.6). For the $l=2 r$-mode $\widetilde{E}$ is given by

$$
\widetilde{E}=\frac{1}{2} \alpha^{2} \Omega^{2} M R^{2} \widetilde{J} .
$$

The time derivative of $\widetilde{E}$ is precisely the quantity that was used to determine the imaginary part of the frequency of the mode in Eq. (2.7):

$$
\frac{d \widetilde{E}}{d t}=-2 \widetilde{E}\left(\frac{1}{\tau_{G R}}+\frac{1}{\tau_{V}}\right) .
$$

Equation (3.12) together with Eq. (3.11) therefore provides a second equation for determining the evolution of the parameters $\Omega$ and $\alpha$ that specify the state of the star:

$$
\Omega \frac{d \alpha}{d t}+\alpha \frac{d \Omega}{d t}=-\alpha \Omega\left(\frac{1}{\tau_{G R}}+\frac{1}{\tau_{V}}\right) .
$$

Equations (3.10) and (3.13) can be combined then to determine the evolution of $\Omega$ and $\alpha$ during the portion of the evolution in which the perturbation remains small:

$$
\begin{aligned}
& \frac{d \Omega}{d t}=-\frac{2 \Omega}{\tau_{V}} \frac{\alpha^{2} Q}{1+\alpha^{2} Q}, \\
& \frac{d \alpha}{d t}=-\frac{\alpha}{\tau_{G R}}-\frac{\alpha}{\tau_{V}} \frac{1-\alpha^{2} Q}{1+\alpha^{2} Q} .
\end{aligned}
$$

The equation of state dependent parameter $Q$ that appears in Eqs. (3.14) and (3.15) is defined by $Q=3 \widetilde{J} / 2 \widetilde{I}$. For the polytropic model considered in detail here $Q=9.40 \times 10^{-2}$. We note that during the initial linear evolution phase the angular velocity of the star $\Omega$ is nearly constant, evolving according to Eq. (3.14) on the viscous dissipation timescale. During this phase the amplitude of the mode $\alpha$ grows exponentially on a timescale that is comparable to the gravitational radiation timescale.

After a short time (about $500 \mathrm{~s}$ in our numerical solutions) the amplitude becomes so large that non-linear effects can no longer be ignored. We have not yet developed the tools needed to follow the evolution exactly during this non-linear phase. However, we do have some intuition about the nonlinear hydrodynamical evolution of gravitationally driven instabilities in rotating stars. This intuition comes from the studies of the effects of gravitational radiation reaction on the evolution of the ellipsoidal models $[15,16]$. In that case the unstable mode grows exponentially until its amplitude is of order unity. At that point a kind of non-linear saturation occurs, and the growth of the mode stops. The excess angular momentum of the star is radiated away and the star evolves toward a new lower angular momentum equilibrium state. We expect a similar situation to pertain in the evolution of the $r$-modes. Thus, we expect non-linear effects will saturate and halt the further growth of the mode when the amplitude of the mode becomes of order unity. Thus, when the amplitude $\alpha$ grows to the value

$$
\alpha^{2}=\kappa,
$$

(where $\kappa$ is a constant of order unity) we stop evolving the star using Eqs. (3.14) and (3.15). Instead we set $d \alpha / d t=0$ during the saturated non-linear phase of the evolution, while continuing to evolve the angular velocity $\Omega$ by Eq. (3.10) as angular momentum is radiated away to infinity by gravitational radiation. During this phase, then the angular velocity evolves by

$$
\frac{d \Omega}{d t}=\frac{2 \Omega}{\tau_{G R}} \frac{\kappa Q}{1-\kappa Q} .
$$

The $r$-mode will evolve during the saturated non-linear phase of its evolution approximately according to Eqs. (3.16) and (3.17). During this phase the star will lose most of its angular momentum, and spin down to a state having an angular velocity that is much smaller than $\Omega_{K} \approx \frac{2}{3} \sqrt{\pi G \bar{\rho}}$. The star will eventually (in about 1 year in our numerical solutions) evolve to a point where the angular velocity and temperature become sufficiently low that the $r$-mode is no longer unstable. The end of the evolution is characterized by a phase in which the viscous forces and gravitational radiation damp out the energy remaining in the mode and move the star slowly to its final equilibrium configuration. During this final phase, the mode is again of small amplitude and so the linear approximation is adequate to describe the evolution. We monitor the quantity on the right side of Eq. (3.15) throughout the non-linear evolution phase. When it becomes negative we change the evolution equations again, from Eqs. (3.16) and (3.17) back to the linear equations Eqs. (3.14) and (3.15).

In summary then, we model the evolution of the $r$-mode as having three distinct phases: (i) The hot young neutron star is born rapidly rotating with a small initial excitation in the $l=2 r$-mode. This mode initially grows exponentially according to Eqs. (3.14) and (3.15). (ii) The amplitude of the mode saturates due to non-linear hydrodynamic effects at a value of order unity. The bulk of the angular momentum of the star is radiated away by gravitational radiation during this phase according to Eqs. (3.16) and (3.17). (iii) The final phase of the evolution begins when the right side of Eq. (3.15) becomes negative so that the mode begins to be damped out. During the final phase the star evolves again according to Eqs. (3.14) and (3.15).

In order to complete our model for the evolution of the $r$-modes we must specify how the temperature of the star evolves with time. We do this by adopting one of the standard descriptions of the cooling of hot young neutron stars. These stars are expected to cool primarily due to the emission of neutrinos via the modified URCA process [17]. The temperature during this phase falls quickly by a simple power law cooling formula [18]:

$$
\frac{T(t)}{10^{9} \mathrm{~K}}=\left[\frac{t}{\tau_{c}}+\left(\frac{10^{9} \mathrm{~K}}{T_{i}}\right)^{6}\right]^{-1 / 6},
$$

where $T_{i}$ is the initial temperature of the neutron star, and $\tau_{c}$ is a parameter that characterizes the cooling rate. For the modified URCA process $\tau_{c} \approx 1 \mathrm{y}$. A typical value for the initial temperature is $T_{i} \approx 10^{11} \mathrm{~K}$. Equation (3.18) can now 


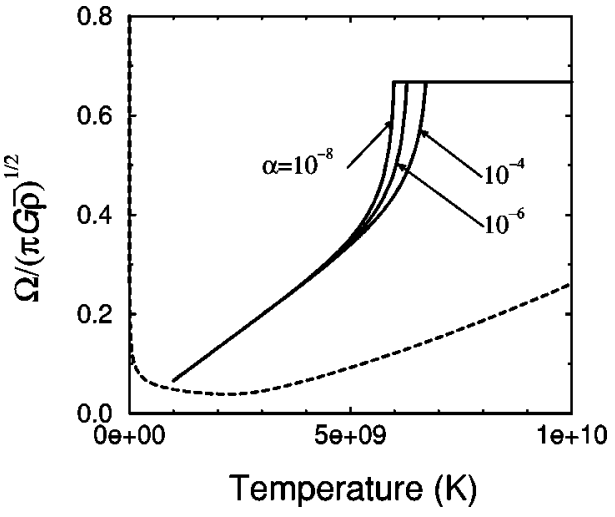

FIG. 1. Angular velocity evolution as gravitational radiation spins down a hot rapidly rotating neutron star. $\alpha$ measures the amplitude of the initial perturbation. The dashed curve shows the critical angular velocity $\Omega_{c}$ above which the $r$-modes are unstable.

be inserted into Eqs. (3.14)-(3.17) to provide explicit differential equations for the time evolution of the angular velocity of the star and the amplitude of the mode. These equations can be solved numerically in a straightforward manner.

Figures 1 and 2 illustrate the solutions to these equations. The dashed curves in Figs. 1 and 2 show the critical angular velocity $\Omega_{c}$, defined by $1 / \tau\left(\Omega_{c}\right)=0$, above which the $r$-modes are unstable. Figure 1 shows the evolution $(\Omega$ plotted versus $T)$ of the angular velocity of the star for $\kappa=1.0$ and a range of values of the initial value of the parameter $\alpha$. In these simulations we have assumed that the initial angular velocity of the star is $\Omega=\Omega_{K}$. This figure illustrates that the final non-linear part of the evolution is remarkably insensitive to the initial size of the perturbation.

Figure 2 illustrates the dependence of the evolution on the parameter $\kappa$ by showing several evolutions with initial values of $\alpha=10^{-6}$. The parameter $\kappa$ measures the degree of saturation that occurs in the non-linear spindown phase of the star. In our numerical studies we examine the limited range $0.25 \leqslant \kappa \leqslant 2$. If $\kappa$ is taken to be too small, the mode simply does not grow to the point that non-linear effects can stop its growth. Conversely if $\kappa$ is taken too large, then our

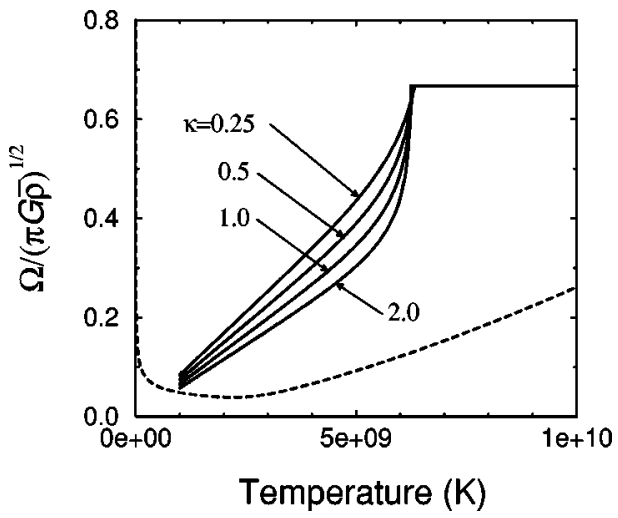

FIG. 2. Evolution of the angular velocity of the star depends on the parameter $\kappa$ in the non-linear saturated phase, but the final angular velocity of the star is insensitive to this. The dashed curve is the same as in Fig. 1. simple evolution equations based in part on the linear perturbation theory become singular, e.g. Eq. (3.17). The equations break down because the (negative) canonical angular momentum of the mode equals the (positive) angular momentum of the equilibrium configuration, and therefore Eq. (3.1) yields the unphysical result that the star has no net angular momentum.

We artificially stop all of our evolution curves when the temperature of the star falls to $10^{9} \mathrm{~K}$. Below this temperature we expect superfluidity and perhaps other non-perfect fluid effects to make our simple simulation highly inaccurate [19]. Figure 2 illustrates that the gravitational radiation instability in the $r$-modes is nevertheless effective in radiating away most of the angular momentum of the star before the star cools to the point that superfluidity or other effects are expected to become important. Figure 2 shows that the amount of angular momentum lost in this process is remarkably insensitive to the value of $\kappa$. Thus, the final upper limit on the angular velocity of the star is (fortunately) fairly insensitive to our assumption about the exact nature of the non-linear portion of the star's evolution.

In this simple model of the evolution of the unstable star, we have ignored the effect that viscous heating might have on the cooling rate of the star. If there were too much viscous heating, then the cooling formula given in Eq. (3.18) would not be correct. We have evaluated the importance of this re-heating effect by comparing the rate at which thermal energy is being radiated away from the star by neutrinos according to Eq. (3.18) with the rate that viscous dissipation deposits thermal energy into the star. Neutrino cooling removes energy from the thermal state of the star at the rate [18]

$$
\frac{d U}{d t}=7.4 \times 10^{39}\left(\frac{T}{10^{9} \mathrm{~K}}\right)^{8} \text { ergs } / \mathrm{s} .
$$

Thermal energy is generated by shear viscosity as the star evolves, but not by bulk viscosity. Bulk viscosity radiates away its excess energy directly by neutrinos without significantly interacting with the thermal energy contained in the star. Thus, energy is transferred from the canonical energy of the $r$-mode to the thermal energy of the star by the formula

$$
\frac{d E_{c}}{d t}=\frac{2 \alpha^{2} \Omega^{2} M R^{2} \widetilde{J}}{\tau_{S}} .
$$

Figure 3 compares the values of $d U / d t$ and $d E_{c} / d t$ for our numerical evolution with $\kappa=1.0$ and initial $\alpha=10^{-6}$. We find that viscosity does not deposit energy into the thermal state of the star at a significant rate until the temperature of the system falls to about $10^{9} \mathrm{~K}$. At this point the star has already lost most of its angular momentum to gravitational radiation, and other dissipative effects (such as those associated with superfluidity), which are not modeled here, will start to play a significant role [19]. Thus, we are justified in ignoring the effects of viscous re-heating on the thermal evolution of the star during the early part of its evolution modeled here. 


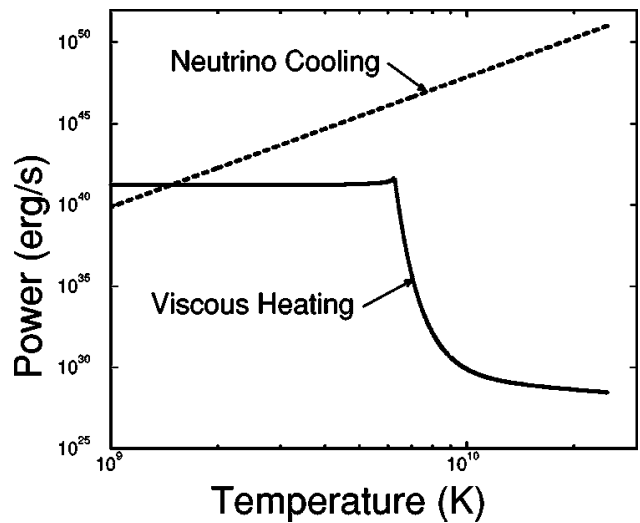

FIG. 3. Cooling rate due to neutrino emission is compared to the viscous heating rate in our numerical evolution of the unstable $r$-modes.

The modified URCA process that determines the thermal evolution used in our evolutions is the standard mechanism by which neutron stars are expected to cool down to about $10^{9} \mathrm{~K}$. Other less standard mechanisms have also been proposed which could significantly speed up the cooling [18]. These mechanisms include neutrino emission processes that require the presence of exotic species (such as quarks or pions) as free particles in the cores of these stars. We have ignored these possibilities in the evolutions described above. If these particles do exist in the cores of neutron stars, we expect that they will only be present in a small volume of material at the centers of these stars. The cores of these stars may well cool rapidly, but the outer layers where the $r$-mode is large will continue to cool at the rate given in Eq. (3.18) until thermal conduction can move energy from the outer layers back into the core.

To estimate what effect a somewhat more rapid cooling might have on the evolution of the $r$-modes, we have artificially varied the value of the parameter $\tau_{c}$ that determines the cooling rate in Eq. (3.18). Figure 4 shows the results of the evolution of an $r$-mode with initial $\alpha=10^{-6}$ and $\kappa$ $=1.0$ for several values of $\tau_{c}$. We see that while the details of the evolution are somewhat affected, the total amount of angular momentum radiated away by gravitational radiation,

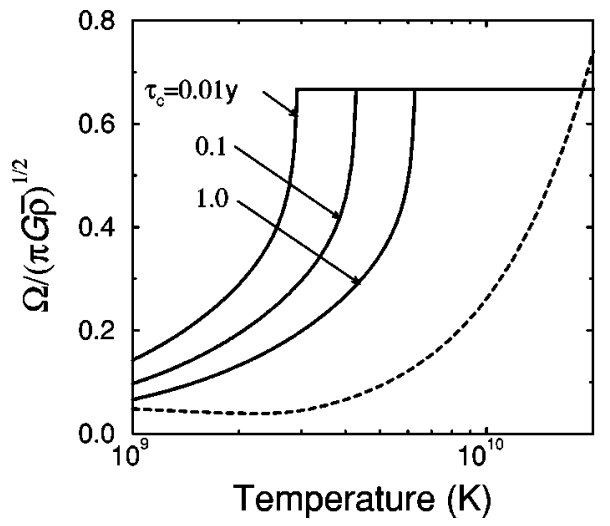

FIG. 4. Angular velocity evolution due to gravitational radiation emission using several values for the timescale $\tau_{c}$ on which the star cools. and the final angular velocity of the star are fairly insensitive to the rate at which the star cools to $10^{9} \mathrm{~K}$.

\section{GRAVITATIONAL WAVEFORMS}

As the $r$-mode grows and evolves it emits gravitational radiation. In this section we calculate the waveforms for the gravitational wave strain $h(t)$ and its Fourier transform $\widetilde{h}(f)$ that are produced by this $r$-mode instability. These are the quantities that can be measured by the gravitational wave detectors now under construction (LIGO, VIRGO, GEO, etc.). During the non-linear saturation phase of the $r$-mode evolution, gravitational radiation controls the dynamics. In this case $\widetilde{h}(f)$ turns out to be independent of the details of the evolution, and thus can be determined quite generally. An evolutionary model is needed, however, to determine $d f / d t$, the time dependence of the various quantities, and the initial and final frequencies of the saturation phase. In this section we present the general model independent derivation of $\widetilde{h}(f)$ for the non-linear saturated phase of the evolution. We also give expressions for the gravitational wave strain that apply to the early phases of the evolution using the simple model discussed in Sec. III.

The frequency-domain gravitational waveform

$$
\widetilde{h}(f) \equiv \int_{-\infty}^{\infty} e^{2 \pi i f t} h(t) d t
$$

is determined completely by the assumption that the angular momentum radiated as gravitational waves comes directly from the angular momentum of the star. This assumption is expected to be satisfied during the non-linear saturated phase of the evolution, but not during the early evolution when the mode is growing exponentially. This derivation is based on Blandford's analysis (as discussed in [20]) of white dwarf collapse to a neutron star which is halted by centrifugal forces (see also [21,22]). Such a star can only collapse to a neutron star by shedding its excess angular momentum through gravitational waves. In that situation as in the nonlinear saturation phase of the $r$-mode evolution, gravitational radiation determines the rate at which angular momentum leaves the system, and this in turn determines the rate at which the frequency of the radiation evolves with time.

In the stationary phase approximation (which is always valid for a secular instability) the gravitational wave strain $h(t)$ is related to its Fourier transform $\widetilde{h}(f)$ by

$$
|h(t)|^{2}=|\widetilde{h}(f)|^{2}\left|\frac{d f}{d t}\right| .
$$

Throughout this discussion we treat $h(t)$ as a complex quantity with purely positive frequency. For the $l=2$ mode of primary importance here, the mode frequency is $\omega=\frac{4}{3} \Omega$, or $f=(2 / 3 \pi) \Omega$, where $f$ is the frequency of the emitted gravitational waves measured in $\mathrm{Hz}$. Assuming the star is uniformly rotating, its angular momentum is $J=I \Omega$, where $I$ is the star's moment of inertia. The moment of inertia is fairly independent of angular velocity (especially at small angular 
velocities where most of the detectable signal from these sources is likely to originate) and is also fairly independent of the amplitude of the excited $r$-mode [see Eq. (3.7)]. Thus, $I$ is reasonably well approximated by its non-rotating value. Thus

$$
\frac{d J}{d f}=\frac{3 \pi}{2} I
$$

The rate at which angular momentum is radiated away by a source is related to the gravitational wave amplitude by the expression [11]

$$
\frac{d J}{d t}=4 \pi D^{2} \frac{m}{\omega} \frac{1}{16 \pi} \omega^{2}\left\langle h_{+}^{2}+h_{\times}^{2}\right\rangle
$$

where $h_{+}$and $h_{\times}$are the amplitudes of the two polarizations of gravitational waves, $D$ is the distance to the source, and $\langle\ldots\rangle$ denotes an average over possible orientations of the source with respect to the observer. Using $d t / d f$ $=d J / d f(d J / d t)^{-1}$ and combining Eqs. (4.2)-(4.4) we obtain

$$
\left\langle\left|\tilde{h}_{+}(f)\right|^{2}+\left|\tilde{h}_{\times}(f)\right|^{2}\right\rangle=\frac{3 I}{4 D^{2} f} .
$$

The measured value of $|\widetilde{h}(f)|^{2}$ depends on the location of the source on the observer's sky as well as its polarization angle with respect to the interferometer. Averaged over these angles, the measured strain is given by

$$
\left\langle|\tilde{h}(f)|^{2}\right\rangle=\frac{1}{5}\left\langle\left|\tilde{h}_{+}(f)\right|^{2}+\left|\tilde{h}_{\times}(f)\right|^{2}\right\rangle .
$$

We are actually interested in the average over source locations in three-dimensional space, not just the two angles on the sky. The spatial average weights more strongly those orientations that yield stronger signals, effectively increasing $\left\langle|\widetilde{h}(f)|^{2}\right\rangle$ by about $1.5[23]$. Combining these results then, the average value of $\tilde{h}$ produced by our fiducial $r$-mode source (with $M=1.4 M_{\odot}, D=20 \mathrm{Mpc}, R=12.5 \mathrm{~km}$ ) is

$$
\tilde{h}(f)=5.7 \times 10^{-25} \sqrt{\frac{1 \mathrm{kHz}}{f}} \mathrm{~Hz}^{-1} \text {. }
$$

Note that this expression does not depend (in the frequency domain) on the details of the evolutionary model apart from the upper and lower frequency cutoffs. This expression, Eq. (4.7), only depends on the assumption that the frequency of the mode evolves as angular momentum is radiated by the star according to Eq. (4.3). We expect this to be satisfied during the non-linear saturated phase of the $r$-mode evolution, but probably not during the early linear growth phase of the mode.

To obtain the complete waveforms for a particular evolutionary model, we start with the usual expression for the gravitational field in terms of its multipoles [11]. We average this expression over angles in the manner described above to obtain

$$
h(t)=\sqrt{\frac{3}{80 \pi}} \frac{\omega^{2} S_{22}}{D} .
$$

For the simple two-parameter evolution of the $r$-mode instability described in Sec. III, this expression can be simplified using Eq. (3.9) to

$$
h(t)=4.4 \times 10^{-24} \alpha\left(\frac{\Omega}{\sqrt{\pi G \bar{\rho}}}\right)^{3}\left(\frac{20 \mathrm{Mpc}}{D}\right) .
$$

This simple evolutionary model also gives a simple formula for the frequency evolution. During the non-linear saturation phase of the evolution Eq. (3.17) can be written as

$$
\frac{d f}{d t} \approx-1.8 \kappa\left(\frac{f}{1 \mathrm{kHz}}\right)^{7} \mathrm{~Hz} / \mathrm{s}
$$

where we have assumed that $\kappa Q \ll 1$. The time for the gravitational wave frequency to evolve to its minimum value $f_{\min }$ is obtained by integrating Eq. (4.10):

$$
t \approx \frac{1.0}{\kappa}\left(\frac{120 \mathrm{~Hz}}{f_{\min }}\right)^{6} \mathrm{y} .
$$

Analogous model dependent expressions can also be derived for the early linear phase of the evolution. During this period the amplitude $\alpha$ grows exponentially on the gravitational timescale according to Eq. (3.14), while the frequency of the mode changes extremely slowly according to Eq. (3.15). Solving these equations approximately gives

$$
\frac{d f}{d t} \approx-\frac{2.7 \alpha^{2}}{t}\left(\frac{f}{1 \mathrm{kHz}}\right)^{3}
$$

Using Eq. (4.9) this implies that $\tilde{h}$ during the linear growth phase is given approximately by

$$
\tilde{h}(f) \approx 4.7 \times 10^{-25} \sqrt{t}\left(\frac{f}{1 \mathrm{kHz}}\right)^{3 / 2} \mathrm{~Hz}^{-1},
$$

where $t \equiv t(f)$ is obtained from Eq. (4.12). The factor $f^{3 / 2}$ that appears in Eq. (4.13) is essentially constant, being given by the initial mode frequency as determined by the initial angular velocity of the star. Since this factor is essentially constant during the linear evolution phase, this implies that $\tilde{h}(f)$ grows as $\sqrt{t}$. The duration of the initial linear growth phase can be estimated from the solution for the amplitude $\alpha$ :

$$
\Delta t=1.5 \times 10^{3}\left(\frac{1 \mathrm{kHz}}{f}\right)^{6} \frac{\ln \left(\sqrt{\kappa} / \alpha_{o}\right)}{\ln \left(10^{6}\right)} \mathrm{s},
$$

where $\alpha_{o}$ is the initial size of the perturbation. During this interval, the mode frequency decreases by an amount of order $0.1 \kappa \mathrm{Hz}$. This is the width of the initial spikes shown in Figs. 5 and 6 . The maximum amplitude achieved by $\tilde{h}$ can also be determined from Eqs. (4.13) and (4.14): 


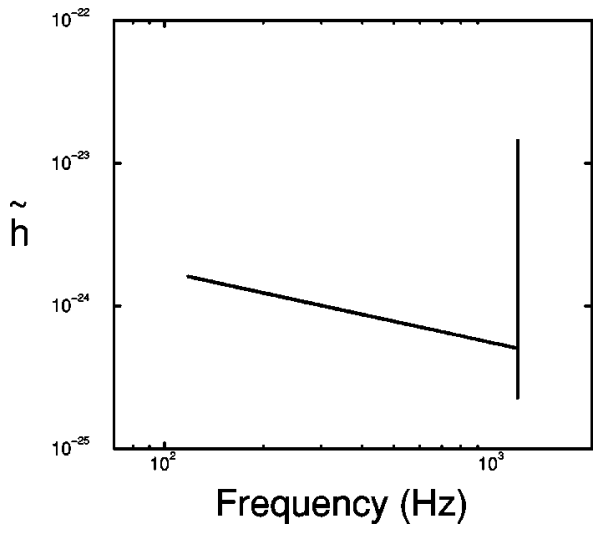

FIG. 5. Frequency dependence of the gravitational wave amplitude $\tilde{h}$ for a source located at $20 \mathrm{Mpc}$.

$$
\max (\tilde{h}) \approx 1.8 \times 10^{-23}\left(\frac{1 \mathrm{kHz}}{f}\right)^{9 / 2}\left[\frac{\log \left(\sqrt{\kappa} / \alpha_{o}\right)}{\log \left(10^{6}\right)}\right]^{1 / 2} \mathrm{~Hz}^{-1}
$$

This expression for $\max (\widetilde{h})$ is fairly insensitive to the duration of the growth phase, and as well to the exact point at which the transition to the non-linear saturation phase occurs. The value of $\max (\tilde{h})$ is rather sensitive to our expression for $d f / d t$, however. If $d f / d t$ were to differ during the linear growth phase from the expression used here by any small effect (such as non-linear modifications of the frequency of the mode) then the the resulting change on $\max (\tilde{h})$ could be large. The analytical expressions given here, Eqs. (4.13)-(4.15), do however accurately represent (to within a few percent) the exact numerical solutions to the equations for our simple model in this region.

Figure 7 illustrates our full numerical solutions for the time dependence of the gravitational wave amplitude $h(t)$ for several values of the parameters $\alpha$ with $\kappa=1$. Figure 8 illustrates the time dependence of $h(t)$ for various values of $\kappa$ with $\alpha=10^{-6}$. All of these curves represent the gravitational radiation emitted by a neutron star initially spinning with angular velocity $\Omega=\Omega_{K}=\frac{2}{3} \sqrt{\pi G \bar{\rho}}$. In this section and the next we terminate the evolution once the star has cooled

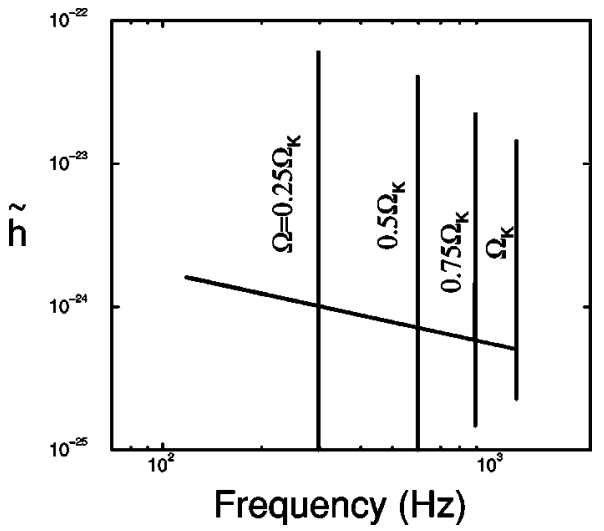

FIG. 6. Frequency dependence of the gravitational wave amplitude $\widetilde{h}$ for various values of the initial angular velocity of the star.

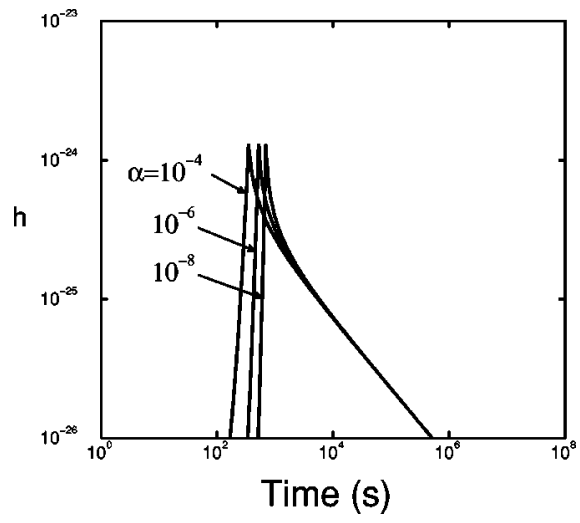

FIG. 7. Time dependence of the gravitational wave amplitude $h(t)$ for a detector located at $D=20 \mathrm{Mpc}$. The peak amplitude is very insensitive to the initial size of the perturbation $\alpha$.

to $10^{9} \mathrm{~K}$. Below this temperature the evolution will be significantly affected by mechanisms not dealt with in this paper, such as the superfluid transition, the re-heating of the star by viscous dissipation in the mode, and dissipation mechanisms (e.g. plate tectonics) associated with the rapidly forming crust.

Figures 5 and 6 illustrate the frequency dependence of $\tilde{h}(f)$ for a $1.4 M_{\odot}$ neutron star located at $20 \mathrm{Mpc}$ using the same values of $\alpha$ and $\kappa$ used in Figs. 7 and 8. Figure 5 illustrates that $\tilde{h}(f)$ is remarkably insensitive to the initial size of the perturbation $\alpha$. The sharp vertical spikes appearing at the high-frequency ends of these curves are due to the extremely monochromatic gravitational waves emitted during the linear growth phase. The structure of this spike in our model is accurately described by Eqs. (4.13)-(4.15), but it is not clear that this spike is a robust feature of our model. During the phase of the evolution that produces the spike, the amplitude of the mode is quite small except for a period of about one minute. Thus the total amount of radiated energy and angular momentum contained in this spike is quite small. The spike is not likely to play an important role in the detection of these sources even if it is a real feature of the $r$-mode instability.

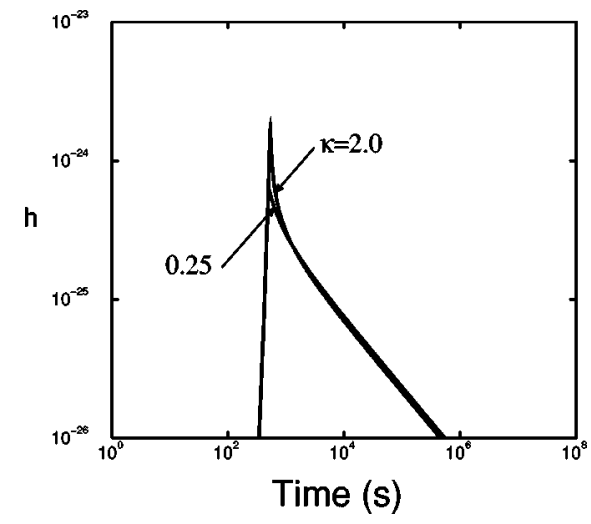

FIG. 8. Time dependence of the gravitational wave amplitude $h(t)$. The late time amplitude is rather insensitive to the nonlinearity parameter $\kappa$. 


\section{DETECTABILITY}

In this section we discuss the detectability of the gravitational radiation emitted by young neutron stars spinning down due to the $r$-mode instability. This radiation might be detected as strong sources from single spindown events or as a stochastic background made up of many weaker sources.

\section{A. Single sources}

First we estimate the signal-to-noise ratio $S / N$ for a single source located at a distance $D$, chosen to be large enough so that there is likely to be a reasonable event rate (say a few events per year). This distance $D$ must be large enough then to include several thousand galaxies (assuming that the observed supernova rate is comparable to the neutron star formation rate). Thus we take this fiducial distance to have the value $D=20 \mathrm{Mpc}$ [24], the approximate distance to the Virgo cluster of galaxies. We estimate the optimal value of $S / N$ that could be obtained by matched filtering. Because matched filtering is probably not feasible for these sources, this estimate provides only an upper limit of what might be achieved. We briefly discuss two more realistic search strategies based on barycentered Fourier transforms of the data [25].

Using matched filtering, the power signal-to-noise ratio $(S / N)^{2}$ of the detection is given by

$$
\left(\frac{S}{N}\right)^{2}=2 \int_{0}^{\infty} \frac{|\widetilde{h}(f)|^{2}}{S_{h}(f)} d f
$$

where $S_{h}(f)$ is the power spectral density of the detector strain noise. The constant in front of the integral in Eq. (4.5) is 2 (instead of 4 as in Ref. [26]) because our $h$ is complex (with purely positive frequency). Equation (5.1) can also be written

$$
\left(\frac{S}{N}\right)^{2}=2 \int \frac{d f}{f}\left(\frac{h_{c}}{h_{\mathrm{rms}}}\right)^{2} .
$$

Here the rms strain noise $h_{\mathrm{rms}}$ in the detector is given by

$$
h_{\mathrm{rms}} \equiv \sqrt{f S_{h}(f)} \text {, }
$$

where $S_{h}(f)$ is the power spectral density of the noise, and the characteristic amplitude $h_{c}$ of the signal is defined by [20]

$$
h_{c} \equiv h \sqrt{f^{2}\left|\frac{d t}{d f}\right|} .
$$

The quantity multiplying $h$ on the right side of Eq. (5.4) is generally interpreted as the number of cycles radiated while the frequency changes by an amount of order $f$. This interpretation is correct as long as the frequency evolution is very smooth. However, our evolutionary model of the frequency evolution contains a discontinuity as the mode stops linearly evolving and saturates. Consequently the actual number of cycles spent near the initial frequency is far fewer than indicated by Eq. (5.4). The quantity $h_{c}$ is a useful estimator of

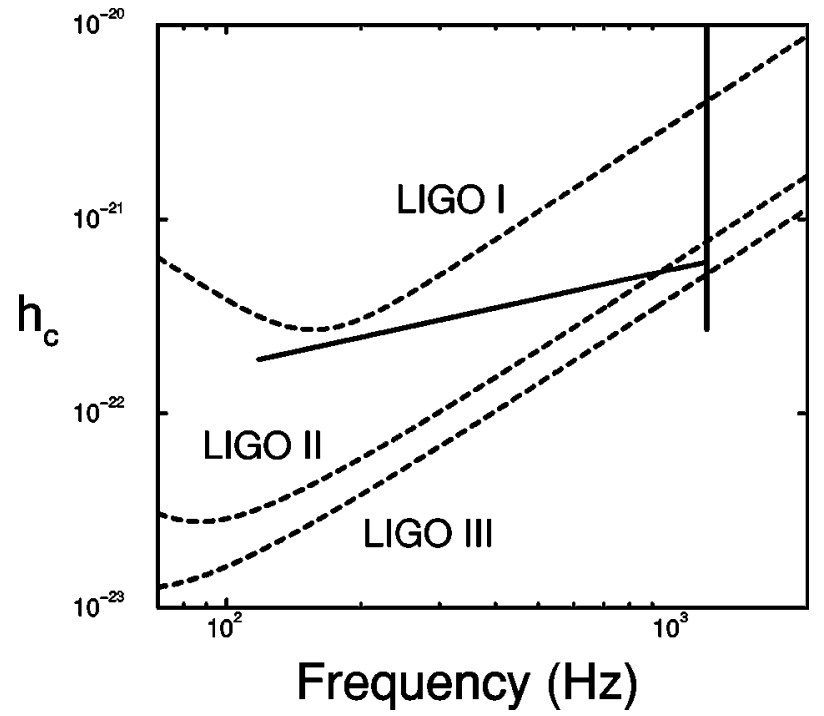

FIG. 9. Characteristic gravitational wave amplitude $h_{c}$ (solid curve) compared to the noise amplitude $h_{\text {rms }}$ (dashed curves) for LIGO.

the effective filtered amplitude of a signal because the integral of $\left(h_{c} / h_{\mathrm{rms}}\right)^{2}$ always gives the optimal $(S / N)^{2}$. Therefore a spike in $h_{c}$ must be interpreted with some cautionthe peak value of the spike is meaningless unless one also knows the bandwidth of the spike.

In Fig. 9 we plot $h_{c}$ versus frequency, superimposed on $h_{\mathrm{rms}}$ for three LIGO interferometer configurations. In the saturation phase (i.e., not including the spike) $h_{c}$ is well approximated by

$$
h_{c} \approx 5.7 \times 10^{-22}\left(\frac{f}{1 \mathrm{kHz}}\right)^{1 / 2} .
$$

We plot $h_{\text {rms }}$ for the LIGO "first interferometers" [5] (which we abbreviate LIGO I), the "enhanced interferometers" [27] (LIGO II), and the "advanced interferometers" [5] (LIGO III). The noise power spectral density for LIGO I is well approximated by the analytical fit [28]

$$
S_{h}(f)=\frac{S_{o}}{3}\left[\left(\frac{f_{o}}{f}\right)^{4}+2\left(\frac{f}{f_{o}}\right)^{2}\right]
$$

where $S_{o}=4.4 \times 10^{-46} \mathrm{~Hz}^{-1}$ and $f_{o}=175 \mathrm{~Hz}$. For LIGO II we construct the approximation

$$
S_{h}(f)=\frac{S_{o}}{11}\left\{2\left(\frac{f_{0}}{f}\right)^{9 / 2}+\frac{9}{2}\left[1+\left(\frac{f}{f_{0}}\right)^{2}\right]\right\}
$$

where $S_{o}=8.0 \times 10^{-48} \mathrm{~Hz}^{-1}$ and $f_{o}=112 \mathrm{~Hz}$. For LIGO III the noise spectral density is well approximated by [29]

$$
S_{h}(f)=\frac{S_{o}}{5}\left\{\left(\frac{f_{0}}{f}\right)^{4}+2\left[1+\left(\frac{f}{f_{0}}\right)^{2}\right]\right\}
$$

where $S_{o}=2.3 \times 10^{-48} \mathrm{~Hz}^{-1}$ and $f_{o}=76 \mathrm{~Hz}$.

Most of the contribution to $S / N$ in Eq. (5.2) comes from the saturation phase of the evolution which is largely model 
independent as discussed earlier. Given a detector noise curve, $S / N$ is thus independent of most details of the waveform except the final frequency of the neutron star. We therefore have

$$
\left(\frac{S}{N}\right)^{2}=\frac{9 I}{10 D^{2}} \int_{f_{\min }}^{f_{\max }} \frac{d f}{f S_{h}(f)} .
$$

The minimum frequency $f_{\min }$ reached by the $r$-mode evolution is about $120 \mathrm{~Hz}$. At frequencies slightly larger than $f_{\min }$, the LIGO II noise is dominated by photon shot noise. If we ignore the other noise components, $S_{h}(f)$ becomes

$$
S_{h}(f)=2.6 \times 10^{-52} f^{2} .
$$

For $f_{\max } \gg f_{\min }$ the integral is dominated by the lower cutoff. Thus, the LIGO II $S / N$ is given approximately by

$$
\frac{S}{N} \approx 8.8\left(\frac{I}{10^{45} \mathrm{cgs}}\right)^{1 / 2}\left(\frac{D}{20 \mathrm{Mpc}}\right)^{-1}\left(\frac{f_{\min }}{120 \mathrm{~Hz}}\right)^{-1} .
$$

Including the other components of the noise decreases $S / N$ somewhat. For the numerical evolutions of our simple model with $\kappa=1.0$ which terminate at $10^{9} \mathrm{~K}$, we find $S / N=1.2$, 7.6, and 10.6 for LIGO I, II, and III, respectively at $D=20 \mathrm{Mpc}$ [30]. These last three results scale with $I$ and $D$ just as in Eq. (5.11), but the dependence on $f_{\min }$ is now more complicated. The contribution to $S / N$ from the high frequency spike in our model is 0.6 for LIGO II, and about 0.1 for LIGO I. While the height of the spike in $h_{c}$ may not be a robust feature of our simple model, the contribution that this spike makes to the overall $S / N$ probably is. These numbers indicate that the gravitational radiation from the $r$-mode instability is a probable source for LIGO II if some nearoptimal data analysis strategy can be developed. It appears unlikely that the radiation from the high frequency spike will be detectable.

Given the recent discovery of the ultrafast young pulsar PSR J0537-6910 in the Large Magellanic Cloud [31], it is interesting to examine the effect on detectability of a relatively high superfluid transition temperature. If the initial period of PSR J0537-6910 was about $7 \mathrm{~ms}$ (as extrapolated from the braking indices of typical young pulsars), that could indicate a superfluid transition temperature of about 2 $\times 10^{9} \mathrm{~K}$ and final gravitational-wave frequency of about 200 Hz. Cutting off our simulations at this point in the evolution, we obtain for LIGO II the $S / N$ of about 5 .

It is clear from Fig. 9 that the first-generation LIGO and VIRGO detectors will not see $r$-mode events from the Virgo cluster. Their sensitivity is a factor of about 8 worse than the enhanced detectors. We have also considered the possibility that GEO [7] might detect these sources by using narrowbanding, where it can improve its sensitivity in a restricted frequency range at the expense of worse sensitivity elsewhere. However, for the kind of broad-spectrum signal produced by the $r$-mode instability, narrow-banding is in fact neutral: the gain of signal-to-noise ratio in the selected band is just compensated by the loss over the rest of the spectrum.

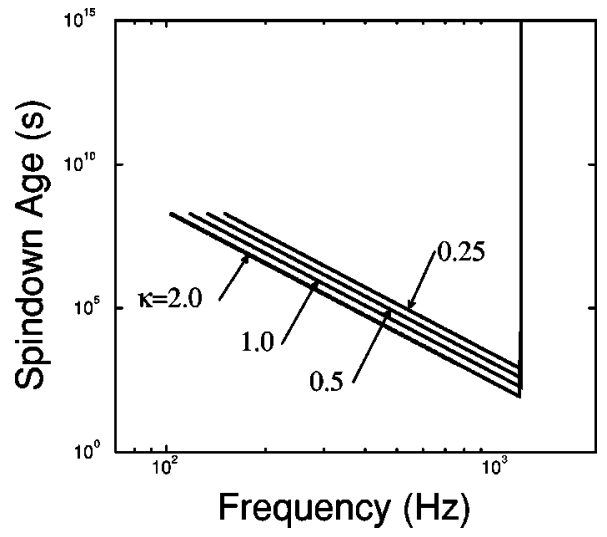

FIG. 10. Spindown age for an $r$-mode-driven young neutron star for different values of the nonlinearity parameter $\kappa$.

So GEO is not likely to see these signals either. Nor will advanced resonant detectors: at their frequencies and in their relatively narrow bandwidths, there is just not enough power in these signals if the sources are in the Virgo cluster. For example, the proposed GRAIL detector [32] operating at its quantum-limited sensitivity $\left(S_{h}=1.6 \times 10^{-48} \mathrm{~Hz}^{-1}\right)$ between 500 and $700 \mathrm{~Hz}$ would have $S / N \approx 1$ for a source at the distance of the Virgo cluster.

Matched filtering using the year-long waveform templates that would be needed to describe these sources completely could yield the signal-to-noise ratios quoted above. However, this is not a practical strategy for this type of signal due to the prohibitively large number of templates that would be needed to parametrize our ignorance of these sources and the resulting high computational cost of filtering the data with these templates. Other strategies equivalent to combining the results of shorter template searches might well be computationally feasible, although they would obtain less than the optimal $S / N$.

The barycentered fast Fourier transform (FFT) technique that has been designed to search for nearly periodic signals [25] might well provide one such method. Figure 10 shows the spindown age

$$
\tau_{\mathrm{sd}}=-f \frac{d t}{d f}
$$

for a neutron star spinning down due to the $r$-mode instability. This quantity provides a reasonably good estimate of the time spent by the evolving star in the saturated non-linear phase, but it is not a good estimate of the amount of time spent during the linear growth phase (for the reasons outlined above). During the saturated phase $\tau_{\mathrm{sd}}$ is given approximately by

$$
\tau_{\mathrm{sd}} \approx \frac{580}{\kappa}\left(\frac{1 \mathrm{kHz}}{f}\right)^{6} \mathrm{~s} \approx \frac{6 t}{\kappa} .
$$

The signal becomes quite monochromatic after about one day, in the sense that the spindown timescale is long compared to the inevitable daily modulation of the signal due to the motion of the Earth. Thus the search techniques for pe- 
riodic sources should work well. In many cases the supernova will be observed, yielding the location of the source and allowing a search over spindown parameters only, a directed spindown search.

The most straightforward way to conduct a directed spindown search is to search over generic spindown parameters as discussed by Brady and Creighton [33]. This involves re-sampling the data so as to render sinusoidal a signal with arbitrary (smooth) frequency evolution, then Fourier transforming it. The signal frequency evolution (which determines the re-sampling) is modeled as

$$
f(t)=f_{0} \sum_{n=0}^{N} \frac{1}{n !}\left(\frac{t}{\tau_{n}}\right)^{n}
$$

where $f_{0}$ is the frequency at the beginning of the FFT, $t$ is the time measured from the beginning of the FFT, and $\tau_{n}$ are expansion parameters with $\tau_{1}=\tau_{\text {sd }}$. The number $N$ of spindown parameters needed is set by the requirement that the frequency drift due to the next term in the series (5.14) be less than one frequency bin of the FFT (which is in turn determined by the integration time $\tau_{\text {int }}$ ). This implies (assuming $\tau_{n} \approx \tau_{\text {sd }}$ ) that

$$
\tau_{\text {int }}^{N+1} \approx \frac{N !}{f_{0}} \tau_{\text {sd }}^{N}
$$

One must choose a set of points in the spindown parameter space for which to perform the re-sampling and FFT. Too few points and one misses signals by re-sampling at the wrong rate; too many and the computational cost of performing all the FFTs becomes prohibitive if data analysis is to be performed "on-line," i.e. keep up with data acquisition. The points in parameter space are chosen using a metric which relates distance in parameter space to loss of $S / N$ [25]. The integration time, which is set so as to optimize the sensitivity of an on-line search for the (fixed) computational power available, is far shorter than a year even for a teraflop computer. Therefore such a search would achieve at best a fraction of the optimal $S / N$ [33]. It is possible that a hierarchical version of this strategy could be developed, in which the best candidates from a year of shorter FFTs are somehow combined to give an improved confidence level. Developing such a strategy would require extensive further investigation.

One way to increase the sensitivity of a directed spindown search would be to constrain the spindown parameter space by taking advantage of whatever information we have about the source from physical models. In practice we do not have and probably will never have completely reliable models for this type of system. The phenomenological model presented here has many physical assumptions that may prove to be inadequate. For instance, in the saturation phase it is highly unlikely that the star can be represented simply as a uniformly rotating equilibrium configuration plus a linear perturbation. When the mode reaches saturation, the mean velocity perturbation is comparable to the rotation rate of the star. Thus in the saturation phase, the velocity field may develop complicated nonlinear structures (such as the cyclones on Jupiter) and possibly contribute to the mass quad- rupole, which might become a significant radiator. Longlived non-axisymmetric structures requiring higher-order modes are seen in the density perturbations of simulations of star formation when there is enough angular momentum [34]. Although the physics is very different in such simulations, a priori we see no reason to believe that the velocity analogues of these structures are not formed by the $r$-mode instability.

However, not all of the physics affects the waveforms. Some kind of phenomenological model of the signal (as opposed to the star) could be enough to substantially reduce the volume of spindown parameter space to be searched. For a reasonable model of the neutron star, all of the terms in the expansion for $d f / d t$ might be determined from a relatively small number of phenomenological parameters. Although quite crude, the model of $r$-mode instability gravitational waveforms provided here has several features which should be fairly robust: the form of $\widetilde{h}(f)$ during the spindown phase, the approximate frequency range of the expected radiation, the approximate timescale for the spindown to occur, etc. Presumably these robust features can be used to reduce considerably the volume of general spindown parameter space which need be searched.

\section{B. Stochastic background}

We now consider the gravitational-wave stochastic background generated by spin-down radiation from neutron star formation throughout the universe. A stochastic background is detected by looking for correlations in the response of two or more detectors. The sensitivity of the network to the background drops rapidly for gravitational wave frequencies much higher than the inverse light-travel time between detectors. For the present application, the important networks are therefore the VIRGO-GEO pair (high frequency cut-off $\left.f_{\text {cut }} \approx 400 \mathrm{~Hz}\right)$ and the Washington-Louisiana pair of LIGO detectors $\left(f_{\text {cut }} \approx 100 \mathrm{~Hz}\right)$. The gravitational radiation generated by the $r$-mode spin-down process has significant power at these relatively low-frequencies, $f \approx 100-400 \mathrm{~Hz}$. In addition the cosmological redshift (with $z \approx 1-4$ ) of these sources has the beneficial effect of shifting much of the radiation into the detectable band.

Neutron stars have presumably been formed since the beginning of star formation. If each neutron star formation event converts a reasonable fraction of a solar mass into gravitational radiation via the $r$-mode instability, then the sum of this radiation constitutes a random background that may be detectable by LIGO III. We now make a rough estimate of the spectrum and detectability of this background radiation. A more detailed analysis is being carried out by Vecchio and Cutler [35].

The spectrum of the gravitational wave background is typically represented by the following dimensionless quantity:

$$
\boldsymbol{\Omega}_{\mathrm{gw}}(f) \equiv \frac{1}{\rho_{c}} \frac{d \rho_{\mathrm{gw}}}{d \ln f},
$$

where $\rho_{\mathrm{gw}}$ is the energy density in gravitational waves, and $\rho_{c}=3 c^{2} H_{0}^{2} / 3 \pi G \approx 1.6 \times 10^{-8} h_{100}^{2} \mathrm{erg} / \mathrm{cm}^{3}$ is the critical en- 
ergy density just needed to close the universe. $\left(H_{0}\right.$ is the Hubble constant and $h_{100}$ is $H_{0}$ divided by $100 \mathrm{~km} / \mathrm{s} / \mathrm{Mpc}$.) The signal-to-noise with which this background can be detected in a correlation experiment between two detectors (here assumed to have uncorrelated noise) is given by $[36,37]$

$$
\left(\frac{S}{N}\right)^{2}=\frac{9 H_{0}^{4} T}{50 \pi^{4}} \int_{0}^{\infty} d f \frac{\gamma^{2}(f) \boldsymbol{\Omega}_{\mathrm{gw}}^{2}(f)}{f^{6}{ }^{1} S_{h}(f){ }^{2} S_{h}(f)},
$$

where $T$ is the integration time, ${ }^{1} S_{h}(f)$ and ${ }^{2} S_{h}(f)$ are the noise spectral densities of the two detectors, and $\gamma(f)$ is the (dimensionless) overlap reduction function, which accounts for the fact that the detectors will typically have different locations and orientations.

We can get a rough estimate of $\boldsymbol{\Omega}_{\mathrm{gw}}(f)$ due to the stochastic background of gravitational radiation from the $r$-mode instability as follows. A $1.4 M_{\odot}$ neutron star rotating with Keplerian angular velocity $\Omega_{K} \approx \frac{2}{3} \sqrt{\pi G \bar{\rho}}$ has rotational kinetic energy $E_{\mathrm{K}} \approx 0.025 M_{\odot} c^{2}$, two-thirds of which is radiated as gravitational waves. For now, assume that all neutron stars are born with angular velocity $\Omega_{K}$. For a single supernova occurring at $z=0$, the spin-down radiation has spectrum $d E_{\mathrm{gw}} / d f \approx \frac{4}{3} E_{\mathrm{K}} f / f_{\max }^{2}$, where $f_{\max } \approx 2 \Omega_{K} / 3 \pi$ $\approx 1400 \mathrm{~Hz}$.

We are chiefly interested in $\boldsymbol{\Omega}_{\mathrm{gw}}(f)$ for $f<f_{\text {min }}$ $\approx 120 \mathrm{~Hz}$, since that is the range where the pair of LIGO III detectors will have their best sensitivity. Let $n(z) d V d z$ be the number of supernovas occurring within co-moving volume $d V$ and redshift interval $d z$. In the frequency range of interest then the spectrum of gravitational radiation in the universe today is given by

$$
\frac{d \rho_{\mathrm{gw}}(f)}{d f} \Delta f=\frac{4 E_{\mathrm{K}}}{3 f_{\max }^{2}} \int_{z_{\min }(f)}^{z_{\max }(f)} n(z) \frac{f^{\prime} \Delta f^{\prime} d z}{1+z},
$$

where $f^{\prime} \Delta f^{\prime}=(1+z)^{2} f \Delta f$ are the values of the frequencies as emitted by the source, $z_{\min }(f) \equiv \max \left\{0, f_{\min } / f-1\right\}, z_{\max }(f)$ $\equiv \min \left\{z_{*}, f_{\max } / f-1\right\}$, and $z_{*}$ corresponds to the maximum redshift where there was significant star formation. The factor $1+z$ in the denominator in Eq. (5.18) accounts for the redshift in energy of the gravitational radiation.

To evaluate the integral in Eq. (5.18), we must make some assumption about the rate of Types Ib, Ic, and II supernovas (which are the ones that leave behind neutron stars). The combined rate in our galaxy at present is roughly one per 100 years. If this rate had been constant, the Galaxy would today contain about $10^{8}$ neutron stars. However at earlier times, for $z$ between 1 and 4 , the rate (with respect to proper time) was significantly higher. A reasonable estimate is that our Galaxy contains $3 \times 10^{8}$ neutron stars today. Let $n(z) \Delta z$ be the density of neutron star births (per unit comoving volume) between redshifts $z$ and $z+\Delta z$. As a rough, first-cut at this problem, we model $n(z)$ as constant $n(z)$ $\equiv n_{o}$ for $0<z<z_{*}$, where $z_{*} \approx 4$, and take $n(z)=0$ for $z$ $>z_{*}$. In this case the integral in Eq. (5.18) can be performed to obtain

$$
\frac{\boldsymbol{\Omega}_{g w}(f)}{A f^{2}}= \begin{cases}0, & 0<f<f_{1}, \\ \left(z_{*}+1\right)^{2}-\left(f_{\min } / f\right)^{2}, & f_{1}<f<f_{\min }, \\ z_{*}\left(z_{*}+2\right), & f_{\min }<f<f_{2}, \\ \left(f_{\max } / f\right)^{2}-1, & f_{2}<f<f_{\max }, \\ 0, & f>f_{\max },\end{cases}
$$

where $A \equiv 2 n_{o} E_{\mathrm{K}} /\left(3 \rho_{c} f_{\text {max }}^{2}\right), \quad f_{1} \equiv f_{\text {min }} /\left(1+z_{*}\right) \quad$ and $\quad f_{2}$ $\equiv f_{\max } /\left(1+z_{*}\right)$.

We can estimate the value of the constant $n_{o}$ as follows. We assume that the number of neutron stars in a given location is roughly proportional to the luminosity of the visible matter at that location. The total luminosity of our Galaxy is $1.4 \times 10^{10} L_{\odot}$ [38], while the number of neutron stars in the Galaxy is about $3 \times 10^{8}$. Thus the neutron star mass to total luminosity of matter ratio is about $0.03 M_{\odot} / L_{\odot}$. The mean luminosity of the universe is $1.0 \times 10^{8} h_{100} L_{\odot} / \mathrm{Mpc}^{3}$ [39]. Thus, the mean mass density of neutron stars is about $\rho_{\mathrm{ns}}$ $\approx 3 \times 10^{6} h_{100} M_{\odot} / \mathrm{Mpc}^{3} \approx 1.1 \times 10^{-5} h_{100}^{-1} \rho_{\mathrm{c}}$. The current density of neutron stars is related to the constant $n_{o}$ by $\rho_{\mathrm{ns}} \approx 1.4 M_{\odot} z_{*} n_{o} \approx 5.6 M_{\odot} n_{o}$. Thus $\frac{2}{3} n_{o} E_{\mathrm{K}} / \rho_{\mathrm{c}} \approx 3.3$ $\times 10^{-8} h_{100}^{-1}$. For LIGO, the most important range in Eq. (5.19) is $f_{1}<f<f_{\min }$; we can re-write the result for this portion in the more useful form

$$
\boldsymbol{\Omega}_{\mathrm{gw}}(f) \approx 2.4 \times 10^{-10} h_{100}^{-1}\left[\left(\frac{f}{24 \mathrm{~Hz}}\right)^{2}-1\right] .
$$

Evaluating at $f=50 \mathrm{~Hz}$, with $h_{100}=0.7$ we find $\boldsymbol{\Omega}_{\mathrm{gw}}(f$ $=50 \mathrm{~Hz}) \approx 1.1 \times 10^{-9}$.

Using the above spectrum Eq. (5.19), we evaluate the $S / N$ using Eq. (5.17). For an integration time of $T=10^{7} \mathrm{~s}$ we find $S / N=0.0022,0.34$, and 2.6 for LIGO I, II, and III respectively [40]. Since there has been some discussion of building a second kilometer-size interferometer in Europe, we also consider the sensitivity to the $r$-mode background of this detector paired with VIRGO. We assume that the detectors will be located less than about $300 \mathrm{~km}$ apart and have the same orientation. [To model this, we simply set $\gamma(f)=1$ in the integral Eq. (5.17).] We find $S / N=5.6$ assuming both these detectors have LIGO II sensitivity, $S / N=0.9$ assuming one detector has LIGO I sensitivity while the other has LIGO III sensitivity, and $S / N=23$ assuming both have LIGO III sensitivity. Thus we see that detection of the $r$-mode background will have to wait either for development of "advanced" interferometers or for the construction of two nearby detectors with "enhanced" sensitivity. Two nearby "advanced" interferometers could see quite a strong signal. All the above results on correlation measurements of the stochastic background assume that magnets will be eliminated from the LIGO design. With the current design long-range correlated magnetic fields from Schuman resonances and lightning strikes will mimic a stochastic background with $\boldsymbol{\Omega}_{\mathrm{gw}}$ approximately $10^{-7}$ to $10^{-9}$ [41].

These calculations assumed that all neutron stars are born with spins near their maximal value $\Omega_{K}$. It should be clear, 
however, that these results for the $S / N$ achievable by the LIGO pair depend only on the stars being born with spins greater than about $300 \mathrm{~Hz}$. Of course, it could well be that some fraction $F$ of neutron stars are born with rapid spins, while $(1-F)$ are born slowly spinning. The values of $S / N$ in this case could be estimated from those given above by multiplying by $F$. (See Spruit and Phinney [42] for a recent argument that most neutron stars should be born with very slow rotation rates.)

It has previously been suggested that there could be a detectable gravitational wave background produced by supernova events $[43,44]$. The stochastic background due gravitational radiation from the $r$-modes differs from that previously envisioned in two important respects. Previously it was assumed that the radiation would be emitted in short bursts, forming a random but not continuous background. For the $r$-mode background, the long duration of the emission guarantees that it will be a continuous hum rather than an occasional pop. Also, the spectrum from spindown radiation extends to lower frequencies than had previously been expected from supernova events.

\section{DISCUSSION}

The discovery of a strong source of gravitational waves that is ubiquitous and is associated with such interesting objects as supernovas and neutron stars would inevitably open up a rich prospect for obtaining astronomical information from gravitational wave observations. We shall discuss here some of the more obvious prospects.

Background radiation from r-modes. Pleasantly, the background requires no detailed modeling of the signal in order to detect it. However, detection of background radiation from $r$-modes will probably have to wait for the development of "advanced" interferometers (or the construction of two nearby "enhanced" interferometers), even if we assume that a large fraction of neutron stars are born rapidly rotating. For nearby detectors with LIGO III sensitivity, $S / N$ is high enough that one could experimentally measure the spectrum $\boldsymbol{\Omega}_{\mathrm{gw}}(f)$ with reasonable accuracy. This might provide very interesting cosmological information. For instance, imagine that most neutron stars are born rapidly rotating, and that observations of nearby supernovas have allowed us to determine $f_{\text {min }}$ (or more likely the distribution function for $f_{\min }$, since differences in masses and post-collapse initial conditions could lead to a range of final angular velocities). Then the background spectrum between 25 and $50 \mathrm{~Hz}$ would give us direct information about the neutron star formation rate in the early universe. Given the neutron star formation rate as a function of redshift, the spectrum between 50 and $\sim 300 \mathrm{~Hz}$ would then tell us about the distribution of initial rotation speeds of neutron stars.

Individual $r$-mode events associated with known supernovas. The easiest case is one where the supernova that leads to the neutron star is seen optically. This gives some idea of when the $r$-mode radiation should be looked for, but more importantly it gives a position. That reduces the difficulty of extracting the signal from the detector data stream. Detection of the radiation will return the amplitude of the signal, its polarization, the final spin of the star, and the values of the parameters of the waveform. Assuming that the three large detectors (the two LIGO installations and VIRGO) all detect the signal with $S / N \approx 8$, the effective combined $S / N$ will be $8 \sqrt{3} \approx 14$. Values of the various parameters will then typically be determined to $10-20 \%$ accuracy. The squared amplitude of the signal, $|\widetilde{h}(f)|^{2}$ is $I / D^{2}$ times a factor that depends on the spin-direction of the neutron star (which, again, should be determined to $10-20 \%$ accuracy from the waveform's polarization). If the host galaxy's distance can be determined to better than the accuracy of the gravitational wave measurement, which seems likely, then this will provide a direct measure of the moment of inertia of a neutron star, to perhaps $20 \%$ accuracy.

There could be several detections per year, which would then shed light on a number of uncertainties. Even if the parameters are only the Taylor expansion coefficients for the frequency, they will constrain models of the $r$-mode spindown. We can expect to get some information about cooling rates, viscosity, crust formation, the equation of state of neutron matter, and the onset of superfluidity (or some combination of these). We also expect variability from event to event, due to different initial conditions after gravitational collapse, such as differential rotation or even the mass of the neutron star. Significant differential rotation might affect the final spin rate of neutron stars; hence any variability in the final spin speed might shed light on these initial conditions.

Using r-mode events as supernova detectors. It may prove possible to create search strategies that are efficient enough to detect $r$-mode spindown even without prior positional information from an optical observation. Then the gravitational wave detectors could become supernova monitors for the Virgo cluster. Some fraction (perhaps as many as half) of the supernovas in Virgo go unnoticed, in thick dust clouds. LIGO and VIRGO would not give optical observers advance notice of the supernova: they will identify a neutron star only a year or so after it was formed. But if discovered, it seems possible that they could locate the position of the event with roughly arc-second accuracy.

This precision would be achieved from the modulation of the signal produced by the motion of the earth [45]. The angular accuracy is similar to that achieved for pulsar observations in the radio. Fundamentally it is the diffraction limit of a gravitational wave telescope with the diameter of the earth's orbit, because the detector acts like a synthesis array as it builds up signal along its orbit around the Sun. The ratio of the gravitational wavelength of about $2000 \mathrm{~km}$ to the Earth's orbital diameter of $2 \mathrm{AU}$ is about $2 \times 10^{-5}$ radians, or $\sim 1$ arcsec. This angular accuracy improves with $S / N$ as well, but it will also be degraded to some degree by uncertainties in the time-evolution of the neutron star spin. If the degradation due to the uncertain spin history turns out to be modest, then detailed follow-up searches for the expanding nebula would be possible, starting perhaps one year after the event. The above scenario assumes, as we have in this paper, that neutron-star cooling takes a year or more. If the alternative cooling scenarios are correct and the star cools in a few days, then the angular accuracy of observations will be very poor. 
Other implications for gravitational radiation from supernovas. The $r$-mode instability also has implications for our expectations of other kinds of gravitational radiation from supernova events. Until now, the relatively slow angular velocities (compared to their maximum possible values) of young neutron stars such as the Crab and Vela pulsars had been an argument that neutron stars were generally born with small angular momentum. The $r$-mode instability essentially eliminates that argument.

It therefore seems to us more likely than before that the gravitational collapse event can also be a strong source of gravitational waves, since it is clear that a collapse that produces a rapidly rotating star will be more likely to radiate strongly. This is especially so if the proto-neutron star can reach the dynamical bar-mode instability that is seen in the lower-density star-formation simulations.

The following scenario seems plausible in at least the extreme cases where rotation completely dominates the last stages of collapse. The collapsed object has so much angular momentum that it forms a bar shape on a dynamical timescale. The object radiates away angular momentum in gravitational waves until the star is finally able to adopt a stable axisymmetric shape. The strong gravitational radiation ceases, to be replaced by the developing $r$-mode radiation. The first burst would be detectable by LIGO II at the distance of the Virgo cluster, and a network of such detectors could give a rough position. This would allow notification of optical astronomers of the event and multi-wavelength follow-up observations. In the gravitational wave data stream, intensive searching for the $r$-mode radiation would follow.

It seems that the recent discovery of the young, 16-ms pulsar, PSR J0537-6910, must impact one's assessment of the likelihood that most neutron stars are born rapidly rotating, and hence of the plausibility of the above scenario. If PSR J0537 was really born with a $7 \mathrm{~ms}$ period, then it was born spinning almost three times faster than the Crab was (extrapolating from the Crab's known age and measured $\dot{P}$ and $\ddot{P}$ ). This could mean that $7 \mathrm{~ms}$ is the limiting period set by the $r$-mode instability, and the Crab was simply born spinning slower than $7 \mathrm{~ms}$. But instead it could mean that PSR J0537 has considerably higher mass than the Crab, and so has a higher limiting angular velocity. Or the two neutron stars could have had different cooling rates, or very different initial amounts of differential rotation, or or for some reason the superfluid damping operated differently in the two cases. Given our uncertainties concerning much of the physics that sets $f_{\min }$, and given that the braking index of PSR J0537 has not yet been measured, it seems to us too early to draw any firm conclusions about its implications.

Summary. Assuming that a reasonable fraction of neutron stars are born rapidly rotating, the $r$-mode instability will slow them down to rotation periods of order $100-150 \mathrm{~Hz}$ (barring the existence of some undiscovered mechanism that damps the $r$-mode), while generating gravitational waves of sufficient strength that they are in principle detectable by LIGO II. However the gravitational waveform will contain about $5 \times 10^{8}$ cycles (assuming the spin down lasts one year), and the observation of these signals will require the construction of gravitational wave templates that maintain the correct phase over most of this stretch of data. Thus it is clear that much work needs to be done to eliminate the uncertainties in our models and to develop effective parametrizations and search algorithms, in order to achieve the signalto-noise that is in principle available.

\section{ACKNOWLEDGMENTS}

We thank Patrick Brady, Jolien Creighton, Teviet Creighton, Scott Hughes, Sterl Phinney, Joseph Romano, and Kip Thorne for helpful discussions. This research was supported by NSF Grant No. AST-9417371 and Grant No. PHY9796079, by the NSF, and by NASA Grant No. NAG5-4093.
[1] N. Andersson, Astrophys. J. (to be published), gr-qc/9706075.

[2] J. L. Friedman and S. M. Morsink, Astrophys. J. (to be published), gr-qc/9706073.

[3] L. Lindblom, B. J. Owen, and S. M. Morsink, Phys. Rev. Lett. 80, 4843 (1998).

[4] N. Andersson, K. D. Kokkotas, and B. F. Schutz, astro-ph/9805225.

[5] A. Abramovici et al., Science 256, 325 (1992).

[6] A. Giazotto, in Gravitational Wave Experiments, edited by E. Coccia, G. Pizzella, and F. Ronga (World Scientific, Singapore, 1995), p. 86.

[7] K. Danzmann et al., in Gravitational Wave Experiments [6], p. 100.

[8] J. Provost, G. Berthomieu, and A. Rocca, Astron. Astrophys. 94, 126 (1981).

[9] J. Papaloizou and J. E. Pringle, Mon. Not. R. Astron. Soc. 182, 423 (1978).
[10] J. Ipser and L. Lindblom (in preparation).

[11] K. S. Thorne, Rev. Mod. Phys. 52, 299 (1980).

[12] J. Ipser and L. Lindblom, Astrophys. J. 373, 213 (1991).

[13] C. Cutler and L. Lindblom, Astrophys. J. 314, 234 (1987); R. F. Sawyer, Phys. Rev. D 39, 3804 (1989).

[14] J. L. Friedman and B. F. Schutz, Astrophys. J. 222, 281 (1978).

[15] S. L. Detweiler and L. Lindblom, Astrophys. J. 213, 193 (1977).

[16] D. Lai and S. L. Shapiro, Astrophys. J. 442, 259 (1995).

[17] We do not consider photon cooling and other processes that determine the cooling in the outer layers of the neutron star because most of the $r$-mode dissipation occurs in the outer mantle, at densities a few times $10^{14} \mathrm{~g} / \mathrm{cm}^{3}$ where neutrino cooling is expected to dominate.

[18] S. L. Shapiro and S. Teukolsky, Black Holes, White Dwarfs, and Neutron Stars (Wiley, New York, 1983). 
[19] L. Lindblom and G. Mendell, Astrophys. J. 444, 805 (1995).

[20] K. S. Thorne, in 300 Years of Gravitation, edited by S. W. Hawking and W. Israel (Cambridge University Press, Cambridge, England, 1987), p. 330.

[21] B. F. Schutz, Class. Quantum Grav. 6, 1761 (1989).

[22] K. S. Thorne, in Compact Stars in Binaries, edited by J. van Paradijs, E. P. J. van den Heuvel, and E. Kuulkers (Kluwer, Dordrecht, 1996), p. 153.

[23] K. S. Thorne (private communication) informs us that this factor, estimated in Ref. [20] as 1.5, can be determined exactly from L. S. Finn and D. F. Chernoff, Phys. Rev. D 47, 2198 (1993) to be about 1.08. This reduces our estimates of $S / N$ by about $15 \%$ - a small change in light of the other uncertainties in our analysis, e.g. the superfluid transition temperature and the large-amplitude behavior of the $r$-modes.

[24] K. S. Thorne, in Theoretical Principles in Astrophysics and Relativity, edited by N. R. Lebovitz, W. H. Reid, and P. O. Vandervoort (University of Chicago Press, Chicago, 1978), p. 149.

[25] P. R. Brady, T. Creighton, C. Cutler, and B. F. Schutz, Phys. Rev. D 57, 2101 (1998).

[26] C. Cutler and É. É. Flanagan, Phys. Rev. D 49, 2658 (1994).

[27] B. Barish et al., LIGO Advanced Research and Development Proposal, Caltech/MIT, unpublished (1996).

[28] K. S. Thorne (private communication).

[29] S. A. Hughes (private communication).

[30] K. S. Thorne (private communication) points out that these $S / N$ are based on the conventional configuration of the LIGO detectors. It might be possible to increase these $S / N$ by moving the "knee" of the photon shot noise upward to about 200 $\mathrm{Hz}$ where more of this signal is produced. This reconfiguration of the detector would simply require changing the reflectivity of some of the corner mirrors.
[31] F. E. Marshall, R. V. Gotthelf, W. Zhang, J. Middleditch, and Q. D. Wang, astro-ph/9803214.

[32] G. Frossati (unpublished).

[33] P. R. Brady and T. Creighton (in preparation).

[34] J. Tohline, J. E. Cazes, and H. S. Cohl, "'The Formation of Common-Envelope, Pre-Main-Sequence Binary Stars,' talk presented by Tohline at the Numerical Astrophysics 1998 Conference, Tokyo, Japan.

[35] A. Vecchio and C. Cutler (in preparation).

[36] É. É. Flanagan, Phys. Rev. D 48, 2389 (1993).

[37] B. Allen, in Relativistic Gravitation and Gravitational Radiation, edited by J. P. Lasota and J. A. Marck (Cambridge University Press, Cambridge, 1997), p. 373.

[38] J. Binney and S. Tremaine, Galactic Dynamics (Princeton University Press, Princeton, 1987).

[39] P. J. E. Peebles, Physical Cosmology (Princeton University Press, Princeton, 1971).

[40] K. S. Thorne (private communication) points out that it may be possible to increase the $S / N$ somewhat by reconfiguration of the detector to adjust the "knee" of the photon shot noise, or by narrowbanding the detectors if they are close together.

[41] B. Allen and J. D. Romano, gr-qc/9710117.

[42] H. Spruit and E. S. Phinney, Nature (to be published), astro-ph/9803201.

[43] D. Blair and L. Ju, Mon. Not. R. Astron. Soc. 283, 648 (1996).

[44] V. Ferrari, in Proceedings of the 12th Italian Conference on General Relativity and Gravitational Physics, edited by M. Bassan et al. (World Scientific, Singapore, 1997), p. 149.

[45] B. F. Schutz, in The Detection of Gravitational Waves, edited by D. Blair (Cambridge University Press, Cambridge, England, 1991), p. 406. 